\title{
Meta-analysis suggests the microbiome responds to Evolve and Resequence experiments in Drosophila melanogaster
}

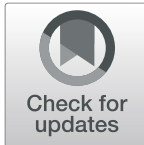

\author{
Lucas P. Henry ${ }^{1,2^{*}}$ (D) and Julien F. Ayroles ${ }^{1,2^{*}}$
}

\begin{abstract}
Background: Experimental evolution has a long history of uncovering fundamental insights into evolutionary processes, but has largely neglected one underappreciated component--the microbiome. As eukaryotic hosts evolve, the microbiome may also respond to selection. However, the microbial contribution to host evolution remains poorly understood. Here, we re-analyzed genomic data to characterize the metagenomes from ten Evolve and Resequence (E\&R) experiments in Drosophila melanogaster to determine how the microbiome changed in response to host selection.

Results: Bacterial diversity was significantly different in 5/10 studies, primarily in traits associated with metabolism or immunity. Duration of selection did not significantly influence bacterial diversity, highlighting the importance of associations with specific host traits.

Conclusions: Our genomic re-analysis suggests the microbiome often responds to host selection; thus, the microbiome may contribute to the response of Drosophila in E\&R experiments. We outline important considerations for incorporating the microbiome into E\&R experiments. The E\&R approach may provide critical insights into hostmicrobiome interactions and fundamental insight into the genomic basis of adaptation.
\end{abstract}

Keywords: Experimental evolution, Drosophila melanogaster, Microbiome

\section{Background}

The microbiome has emerged as a key modulator of many organismal phenotypes [1-3]. While many studies show the impact of the microbiome on host phenotypes, the evolutionary implications remain enigmatic [4-6]. The microbiome may contribute to host evolution in unique ways. First, large effective population sizes and rapid generation times may enable microbes to evolve more rapidly than hosts [7]. Second, the microbiome likely encodes distinct genes compared to the host genome, potentially expanding the genomic reservoir to enable adaptation to diverse selective pressures $[3,8,9]$. If

\footnotetext{
*Correspondence: Ihenry@princeton.edu; jayroles@princeton.edu 'Department of Ecology \& Evolutionary Biology, 150 Carl Icahn Laboratory, Princeton University, Princeton, NJ 08544, USA

Full list of author information is available at the end of the article
}

hosts can leverage this microbial evolution, then the microbiome may alter host evolution.

Experimental evolution is a powerful tool to study the basis of adaptation, but remains underutilized in the study of host-microbiome evolution $[6,10,11]$. One particularly well-suited class of these studies is Evolve and Resequence (E\&R) experiments [12-14]. E\&R experiments build on the long history of using artificial selection in evolutionary biology by incorporating new advances in sequencing technologies to measure the genomic responses to selection. E\&R experiments are commonly performed in microbes like $E$. coli or yeast, as well as eukaryotes like Drosophila [13]. In general, E\&R experiments begin with large outbred populations. The population is reared under a particular selective regime. The selective regime can take many forms, ranging from

(c) The Author(s). 2021 Open Access This article is licensed under a Creative Commons Attribution 4.0 International License, which permits use, sharing, adaptation, distribution and reproduction in any medium or format, as long as you give appropriate credit to the original author(s) and the source, provide a link to the Creative Commons licence, and indicate if changes were made. The images or other third party material in this article are included in the article's Creative Commons licence, unless indicated otherwise in a credit line to the material. If material is not included in the article's Creative Commons licence and your intended use is not permitted by statutory regulation or exceeds the permitted use, you will need to obtain permission directly from the copyright holder. To view a copy of this licence, visit http://creativecommons.org/licenses/by/4.0/ The Creative Commons Public Domain Dedication waiver (http://creativecommons.org/publicdomain/zero/1.0/) applies to the data made available in this article, unless otherwise stated in a credit line to the data. 
threshold selection (e.g., egg size) or general survival under some sort of stressor (e.g., low nutrition diets). In parallel, to control for genetic drift, control populations are maintained in a benign (i.e., non-selective) environment. After a number of generations, the control and evolved populations are sequenced to identify regions of the genomes associated with the response to selection. For flies and other eukaryotic hosts, selection is explicitly applied to host populations, but may also act upon the microbiome. When the microbiome influences host phenotypic variation, microbial variation may also affect the response to selection in hosts. Thus, the underappreciated interplay between host and microbial variation has the potential to complicate the interpretation of selection responses based strictly on host genetic variation.

Microbes may be underappreciated drivers of host phenotypic variation. For example, Wolbachia infection can rescue deleterious phenotypes in homozygous mutant Drosophila lines [15-17]. Body color in aphids is partially determined by Rickettsia secondary symbionts [18]. These phenotypic effects are not limited to single microbial species, but also include more complex microbiomes. In cows, the microbiome explained $13 \%$ of methane emissions [19] and $26-42 \%$ of fatty acid composition of milk [20]. The microbiome also explained $33 \%$ of weight gain in pigs [21]. For both pigs and cows, the microbiome contributed almost as much to traits as host genetics. These examples suggest that the microbiome in many host taxa is an important determinant in host phenotypes, and, in turn, may shape the selection response for hosts. E\&R experiments may thus be missing a substantial component that shapes the host evolutionary response.

Here, we analyzed the metagenomes from 10 E\&R experiments in Drosophila melanogaster. Many phenotypes in D. melanogaster are responsive to microbial variation, including developmental, metabolic, and immunological traits [22-24]. Furthermore, E\&R experiments in D. melanogaster capture the evolutionary response to a wide range of different selective pressures, ranging from life history to nutritional to pathogen challenges (Table 1). Thus, E\& $\mathrm{R}$ experiments in $D$. melanogaster provide a unique opportunity to study how the microbiome responds to host selection. Our goal here is to explore these publicly available data and using meta-analysis and characterize patterns in the metagenomes of these

Table 1 Evolve \& Resequence studies analyzed

\begin{tabular}{|c|c|c|c|c|}
\hline Pressure & Evolved Phenotype & $\begin{array}{l}\text { Duration of } \\
\text { Selection } \\
\text { (generations) }\end{array}$ & $\begin{array}{l}\text { Wolbachia } \\
\text { (\% reads, } \\
\text { min-max) }\end{array}$ & Experimental design (D: diet, S: sequencing) \\
\hline $\begin{array}{l}\text { Accelerated } \\
\text { development } \\
{[25]}\end{array}$ & $\begin{array}{l}\text { Flies developed from egg to } \\
\text { adult 20\% faster than control }\end{array}$ & 605 & $\begin{array}{l}\text { Infected } \\
(0.01-1.40 \%)\end{array}$ & $\begin{array}{l}\text { D: Banana, corn syrup, agar. S: } 25 \text { females (age not reported) } \\
\text { pooled from each line; } 4 \text { control and } 4 \text { evolved. }\end{array}$ \\
\hline $\begin{array}{l}\text { Delayed } \\
\text { reproduction } \\
{[26]}\end{array}$ & $\begin{array}{l}\text { Age of reproduction increased } \\
\text { from } 28 \text { to } 40 \text { days }\end{array}$ & 50 & $\begin{array}{l}\text { Uninfected } \\
(0 \%)\end{array}$ & $\begin{array}{l}\text { D: Not specified. S: } 100 \text { females (age not reported) pooled from } \\
\text { each line; } 18 \text { control and } 18 \text { evolved. }\end{array}$ \\
\hline $\begin{array}{l}\text { Increased } \\
\text { lifespan [27] }\end{array}$ & $\begin{array}{l}\text { Median lifespan was increased } \\
\text { from } 4 \text { weeks to } 7-8 \text { weeks }\end{array}$ & 48 & $\begin{array}{l}\text { Uninfected } \\
(0 \%)\end{array}$ & $\begin{array}{l}\text { D: Agar, yeast, sugar, oatmeal. S: } 250 \text { males }+250 \text { females (age } \\
\text { not reported) pooled from each line; } 3 \text { control and } 3 \text { evolved. }\end{array}$ \\
\hline Egg size $[28]$ & $\begin{array}{l}\text { Egg size was selected } \sim 20 \% \\
\text { larger and smaller eggs }\end{array}$ & 16 & $\begin{array}{l}\text { Infected } \\
(0.02-0.11 \%)\end{array}$ & $\begin{array}{l}\text { D: Not specified. S: } 100 \text { females (age not reported) pooled from } \\
\text { each line; } 3 \text { control, } 3 \text { small, } 3 \text { large. }\end{array}$ \\
\hline $\begin{array}{l}\text { Desiccation } \\
\text { resistance } \\
{[29]}\end{array}$ & $\begin{array}{l}\text { Desiccation resistance (hrs } \\
\text { until } 80 \% \text { mortality) increased } \\
70-80 \%\end{array}$ & 48 & $\begin{array}{l}\text { Infected } \\
(70.2-89.7 \%)\end{array}$ & $\begin{array}{l}\text { D: Yeast, cornmeal, agar. S: } 100 \text { females (age not reported) } \\
\text { pooled from each line; } 3 \text { control and } 3 \text { evolved. }\end{array}$ \\
\hline $\begin{array}{l}\text { Fluctuating } \\
\text { temperature } \\
{[30]}\end{array}$ & $\begin{array}{l}\text { Survival under fluctuating } \\
\text { temps } 18-28^{\circ} \mathrm{C} \text { daily }\end{array}$ & 37 & $\begin{array}{l}\text { Infected } \\
(48.7-75.1 \%)\end{array}$ & $\begin{array}{l}\text { D: Standard media. S: } 500 \text { females ( } 7 \text { days old) pooled for each } \\
\text { line at different time points; beginning, middle, and end; } 3 \\
\text { control and } 3 \text { evolved--only compared beginning and end. }\end{array}$ \\
\hline $\begin{array}{l}\text { Salt }+ \\
\text { cadmium } \\
\text { resistance } \\
{[31]}\end{array}$ & $\begin{array}{l}\text { Survival in constant, spatially, } \\
\text { temporally varying salt and/or } \\
\text { cadmium }\end{array}$ & 42 & $\begin{array}{l}\text { Infected } \\
(0.01-0.03 \%)\end{array}$ & $\begin{array}{l}\text { D: Yeast, cornmeal, sugar agar. Diet differed between control and } \\
\text { evolved. S: } 70 \text { females (age not reported) pooled from each line; } \\
3 \text { control lines and } 5 \text { lines for each selection pressure. }\end{array}$ \\
\hline $\begin{array}{l}\text { Starvation } \\
\text { resistance } \\
{[32]}\end{array}$ & $\begin{array}{l}\text { Starvation resistance (hrs to } \\
\text { death w/o food) increased } \sim \\
25 \%\end{array}$ & 83 & $\begin{array}{l}\text { Infected } \\
(53.0-78.4 \%)\end{array}$ & $\begin{array}{l}\text { D: Not specified. S: } 100 \text { females ( } 4 \text { days old) pooled for each line; } \\
3 \text { control and } 3 \text { evolved lines. }\end{array}$ \\
\hline $\begin{array}{l}\text { Parasitoid } \\
\text { resistance } \\
\text { [33] }\end{array}$ & $\begin{array}{l}\text { Resistance to parasitoid } \\
\text { increased from } 20 \text { to } 50 \%\end{array}$ & 5 & $\begin{array}{l}\text { Uninfected } \\
(0 \%)\end{array}$ & $\begin{array}{l}\text { D: Not specified. S: } 50 \text { females ( } ~ 5 \text { h old) pooled from each line; } \\
16 \text { control and } 16 \text { evolved lines. }\end{array}$ \\
\hline $\begin{array}{l}\text { Viral } \\
\text { resistance } \\
{[34]}\end{array}$ & $\begin{array}{l}\text { Resistance to Drosophila C } \\
\text { virus increased from } 25 \text { to } 75 \%\end{array}$ & 20 & $\begin{array}{l}\text { Infected } \\
(95.1-98.1 \%)\end{array}$ & $\begin{array}{l}\text { D: Standard cornmeal-agar. S: } 200 \text { individuals (age not reported) } \\
\text { pooled from each line; } 4 \text { control, } 4 \text { procedure control, } 4 \text { evolved. }\end{array}$ \\
\hline
\end{tabular}


experiments. This meta-analysis allowed us to identify common patterns in the response of the microbiome to host selection. We use these observations to highlight potential future directions for which the powerful E\&R approach is uniquely suited to identify signatures of selection in host-microbiome evolution.

\section{Results}

The 10 E\&R experiments analyzed for metagenomes ranged in a variety of selective pressures (see Table 1 for full description)--from life history (accelerated development [25], delayed reproduction [26], increased lifespan [27], egg size [28]) to abiotic pressures (desiccation resistance [29], fluctuating temperature [30], salt and heavy metal resistance [31]) to biotic pressures (starvation resistance [32], parasitoid resistance [33], viral resistance [34]). All experiments had replicated control and evolved populations, although replication varied from as few as three to as many as 18 (Table 1). Given the importance of the diet in shaping microbial variation, we examined the reported characteristics of the diet from each study. Importantly, for $9 / 10$ studies, the reported diets did not differ between control and evolved populations (Table 1). Only for the cadmium and salt resistance study [31] were diets different for the entire lifespan between control and evolved populations. The starvation resistance study [32] exerted starvation on adults for 4 days, and then flies that survived were returned to a standard diet to propagate the next generation. While the diets may have varied between studies, only 5/10 studies described the diet (Table 1 ). The lack of consistent dietary reporting is a major challenge for Drosophila-microbiome studies [35]. As the majority of these studies do not report specific dietary information, we are unable to explore the effects of diet across E\&Rs in this analysis.

Because each experiment has replicated control and evolved populations, we compared microbiomes within each experiment. In the E\&R context, control populations represent the standing genetic variation from which selection proceeds. Thus, by comparing control and evolved populations within experiment, the effects of many different factors (e.g., local laboratory environment, different diets, different fly populations) are controlled for in our analysis. For each experiment, bacterial families were differentially abundant in control and evolved populations (Fig. 1; Supp. Figs 1-10 for individual replicates for each experiment). Control and evolved populations tended to harbor similar bacterial families across replicates, within each experiment, as measured through beta-diversity (Jaccard similarity; Fig. 2, Table 2). Only in two experiments did control and evolved populations differ in community membership--accelerated development time and delayed reproduction. Bacterial alpha-diversity frequently responded to experimental evolution (Fig. 3). Evolved populations often exhibited reduced levels of bacterial diversity (4/10 studies), though in one case (accelerated development time) bacterial diversity increased (Table 3 for statistical summary). Taken together, the microbiome frequently shifts in response to host selection (i.e., differences in alphadiversity), but does not necessarily gain different microbes (i.e., no difference in beta-diversity).

Because the number of generations varied across E\&R experiments (from 5 to 605 Drosophila generations; see Table 1), we also tested if change in microbial diversity was correlated with duration of host selection. One hypothesis is that shorter selection experiments provide less opportunity for the microbiome to change, while longer selection provides more opportunity for increased microbial change. The change in microbial diversity was not correlated with duration of selection after controlling for each study as a random effect (Fig. 4, $r=0.05$, $p=0.649$ ). The specific nature of the selective pressure appears to be more important in driving changes in the evolving microbiome as experiment explained $76 \%$ of variance in our model (Table 4). For example, the evolved microbiome in the starvation resistance experiment exhibited the greatest change in bacterial diversity. This may not be surprising given that the Drosophila microbiome has been shown to be tightly linked to the regulation of metabolic networks [22]. For other traits, like egg size, the microbiome did not significantly respond to experimental evolution. This analysis suggests that the effect of selection on microbiome is likely trait specific.

We note that, in seven out of ten studies, flies were infected with Wolbachia (Table 1). Wolbachia was $<2 \%$ of reads for three studies, but $48-98 \%$ for the other four studies. To better understand the association between Wolbachia and the microbial response to selection, we focused on these four studies with high relative abundance (Fig. 5). Wolbachia was significantly more abundant for evolved populations in starvation and viral resistance, though also tended to increase for desiccation resistance and fluctuating temperatures (Table 5 for statistical summary). Taken together, these results highlight how Wolbachia may underlie some of the significant changes to diversity that occur in evolved populations.

\section{Discussion}

To our knowledge, this is the first systematic examination of the microbiome in E\&R experiments in D. melanogaster. Given the many fundamental insights gained from Drosophila in E\&R experiments [13], our results here uncover another layer of variation previously unexplored--the microbiome. The microbiome changed under some selective pressures, while it was unaffected 


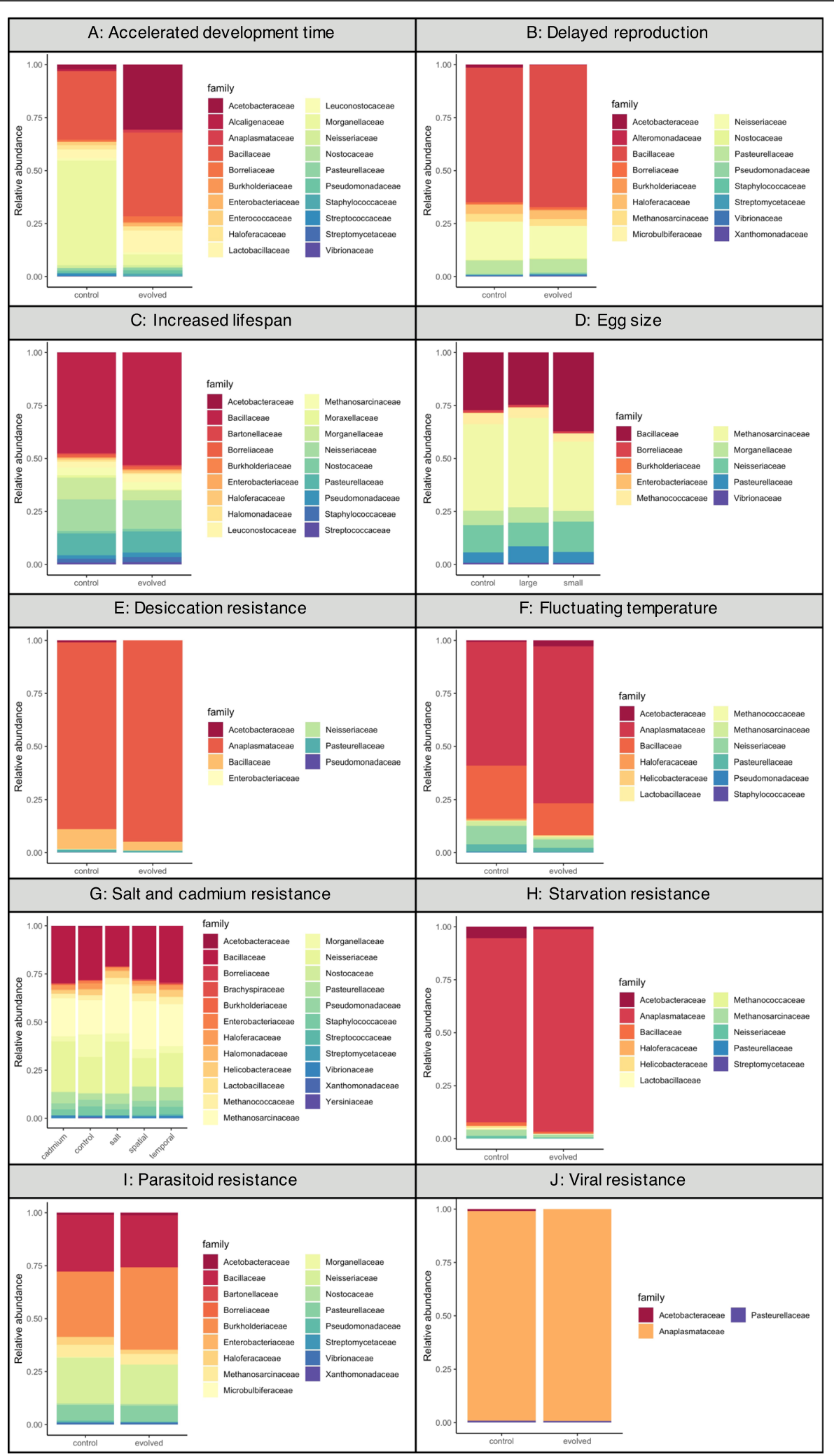

Fig. 1 Relative abundance for bacterial families from the 10 E\&R experiments. Each experiment was grouped separately; the colors represent different bacterial families in each. Only bacteria that comprised $>1 \%$ of total reads were visualized 


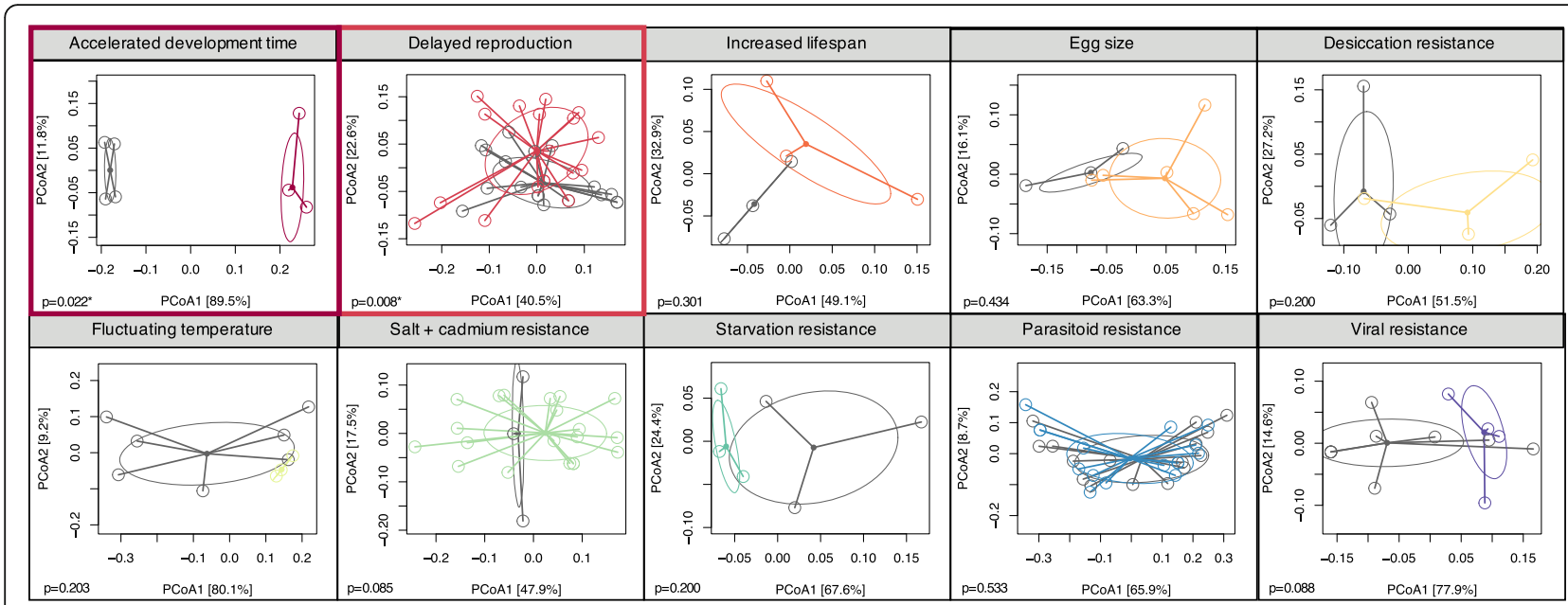

Fig. 2 PCoA plots for beta-diversity using Jaccard similarity for the 10 E\&R experiments show the majority of control and evolved populations harbor similar bacterial families. Each experiment was grouped separately; grey color represents control populations, and the colored points represent evolved populations. Each sequenced pool is shown as a point with lines connecting to the centroid based on PCoA clustering. The two studies (accelerated development time and delayed reproduction) with significantly divergent microbiomes between control and evolved populations are outlined in colored boxes. P-values are shown for all studies

by others (Figs. 1 and 3). Pressures closely linked to metabolic processes, like starvation resistance or development time, or immunity affected microbial diversity the most. In Drosophila, bacterial genes that increase glucose assimilation and fat storage are necessary for bacterial establishment in the host gut, suggesting that hosts select for bacteria to enhance metabolism [36-38]. Other pressures, like selection for increased lifespan, egg size, or abiotic stressors (e.g temperature and heavy metals), did not substantially impact microbial diversity (Fig. 3). It is not surprising that not all selection pressures shape the microbiome; indeed, in Drosophila, traits such as activity level, sleep, and some aspects of nutrition are known to not be influenced by the microbiome [39-42]. Our results suggest that the microbiome changes along with host evolution in the E\&R context although we emphasize that the data we present are a re-analysis of existing genomic data and not derived from new manipulative experiments. Our results here contribute to a growing body of literature suggesting that when the microbiome contributes to host phenotypic variation, changes in the microbiome have the potential to impact host evolutionary trajectories $[6,43]$. The evolutionary interplay between host and their microbiome may play an important role in driving host evolution (beyond the explicit selective pressures exerted in these experiments), and this level of variation should not be ignored in $E \& R$ analyses.

We observed several generalities in the microbial response in E\&R experiments. First, the microbiome in both control and evolved populations was composed of similar bacterial families (Fig. 1), suggesting selection did not lead to the complete replacement by different bacterial taxa in evolved populations. In evolved populations, only a few of the bacterial families increased in relative abundance. Furthermore, in all studies, replicate lines from both control and evolved populations show similar community compositions, suggesting consistent effects on the microbiome (Fig. 2, Supp. Figs. 1-10). Because community composition is consistent across replicates, we do not believe drift explains the observed reduction in diversity. If drift was a predominant force shaping the microbiome in evolved populations, we would have expected stochasticity to increase diversity across replicates within control and evolved populations. More likely, bacteria that contribute to the host adaptation may be more likely to persist under the selective pressure, increasing in abundance and facilitating local adaptation. Second, the increase in abundance of particular bacterial families, like Wolbachia, also contributed to the frequent reduction in diversity. The reduction in diversity likely reflects local adaptation in the microbiome, but potentially also the loss of genetic diversity in the host. We expect that the rapid nature of E\&R experiments, combined with strong selective pressures, results in lower heterozygosity levels across the genome following selection in E\&R experiments [13]. Host genetics shapes a significant fraction of the fly microbiome [44], and perhaps the loss of diversity in the host genome also contributed to the reduction in microbial diversity observed here. While evolved populations have reduced heterozygosity, they still maintain substantial heterozygosity across the genome. More research is necessary to understand how host genome-wide diversity affects microbial diversity, or if only certain host loci are the key drivers of changes in microbial diversity. 
Table 2 Summary statistics for beta-diversity (Jaccard similarity)

\section{Accelerated development time}

$\begin{array}{lll} & \text { Df } & \text { SumsOfSqs } \\ \text { group } & 1 & 0.30394 \\ \text { Residuals } & 5 & 0.03719 \\ \text { Total } & 6 & 0.34113\end{array}$

\section{Delayed reproduction}

$\begin{array}{lll} & \text { Df } & \text { SumsOfSqs } \\ \text { group } & 1 & 0.09902 \\ \text { Residuals } & 34 & 0.86288 \\ \text { Total } & 35 & 0.9619\end{array}$

Increased lifespan

$\begin{array}{lll} & \text { Df } & \text { Sum Sq } \\ \text { Groups } & 1 & 0.0031624 \\ \text { Residuals } & 4 & 0.0068525\end{array}$

Egg size

Groups

Desiccation resistance

$\begin{array}{ll} & \text { Df } \\ \text { group } & 1 \\ \text { Residuals } & 4 \\ \text { Total } & 5\end{array}$

Fluctuating temperature

$\begin{array}{ll} & \text { Df } \\ \text { group } & 1 \\ \text { Residuals } & 8 \\ \text { Total } & 9 \\ \text { Salt + cadmium resistance }\end{array}$

\section{Salt + cadmium resistance}

$\begin{array}{lll} & \text { Df } & \text { SumsOfSqs } \\ \text { group } & 1 & 0.05652 \\ \text { Residuals } & 21 & 0.56081 \\ \text { Total } & 22 & 0.61733\end{array}$

\section{Starvation resistance}

group
Residuals
Total

Parasitoid resistance

$\begin{array}{lll} & \text { Df } & \text { SumsOfSqs } \\ \text { group } & 1 & 0.04295 \\ \text { Residuals } & 30 & 1.99099 \\ \text { Total } & 31 & 2.03394\end{array}$

\section{Viral resistance}

MeanSqs
0.303937
0.007438
MeanSqs
0.099022
0.025379

Mean Sq

0.0031624

0.0017131
Mean Sq

0.0020672

0.0027276

MeanSqs

0.044141

0.023262

0.137188

SumsOfSqs
0.11007
0.44424
0.55432

SumsOfSqs

0.019872

0.037964

0.057836

MeanSqs

0.019872

0.009491

Df

MeanSqs

0.11008

0.05553

MeanSqs

0.056519

0.026705

MeanSqs

0.04295

0.066366

MeanSqs
F.Model

2.1164

F.Model

1.9823

F.Model

1.8976

0.32176

0.67824

1

$\mathrm{R}^{2}$

0.19858

0.80142

1

$\mathrm{R}^{2}$

0.09155

0.90845

1

F.Model

2.0937

$\mathrm{R}^{2}$

0.34359

0.65641

1

F.Model

0.64716

$\mathrm{R} 2^{2}$

$\operatorname{Pr}(>\mathrm{F})$

0.02112

0.97888

1

0.2

.

$\operatorname{Pr}(>\mathrm{F})$

0.203

$\operatorname{Pr}(>\mathrm{F})$

0.085

$\operatorname{Pr}(>\mathrm{F})$

0.2

0.533

F.Model

$\mathrm{R} 2^{2}$

$\operatorname{Pr}(>\mathrm{F})$
$\operatorname{Pr}(>\mathrm{F})$

$0.022^{*}$

0.10902

1

$\operatorname{Pr}(>\mathrm{F})$

$0.008^{* *}$

0.10294
0.89706

(1)

.Perm

$\operatorname{Pr}(>\mathrm{F})$

0.3014

$\operatorname{Pr}(>\mathrm{F})$

0.434

$\operatorname{Pr}(>\mathrm{F})$ 
Table 2 Summary statistics for beta-diversity (Jaccard similarity) (Continued)

\begin{tabular}{llllll}
\hline group & 1 & 0.0384 & 0.0384 & 2.673 & 0.21092 \\
Residuals & 10 & 0.14366 & 0.014366 & & 0.78908 \\
Total & 11 & 0.18206 & & 1
\end{tabular}

Uncovering the specific host genetic loci that may be associated with microbial changes is beyond the scope of this current study; however this is an important factor to consider in future studies.

For the evolved microbiomes, bacteria may have evolved different functions that hosts can leverage. For example, the relative abundance of Acetobacteraceae is enriched in the evolved populations for accelerated development time (Fig. 1a). Acetobacter produces acetic acid that modulates the insulin/insulin-like signaling (IIS) growth factor pathway in flies [36]. More so, Acetobacter is frequently associated with accelerated development compared to other bacteria [24, 36, 45, 46]. The IIS pathway may also integrate metabolic products from other bacteria in the microbiome to help regulate fly metabolism. Wolbachia infection has been shown to increase insulin signaling in Drosophila [17]. The increased Anaplasmataceae abundance in the evolved populations may better regulate metabolic traits to mitigate selection in the starvation resistance experiment (Fig. 1h). We hypothesize that increased relative abundance for particular bacteria in the evolved populations corresponds to functional changes and is suggestive of fitness benefits for the fly. Subsequently, flies transmit and preferentially associate with the beneficial microbes. However, bacteria may also be increasing in the evolved conditions independently of any host selection. To better understand how microbial evolution interacts with host evolution, longitudinal sampling over the course of the evolutionary trajectory is necessary. Identifying if beneficial adaptations emerge first in the microbiome and then alter allele frequencies in host populations would provide key insights into how hostmicrobiome interactions shape eukaryotic evolutionary processes.

The temporal aspect of host-microbiome evolution is important, but underexplored and thus poorly understood. Our analysis suggests that time did not significantly affect the difference in diversity between control and evolved populations (Fig. 4). This might be because the microbiome changes rapidly, within a single host generation, but the evidence for rapid change is

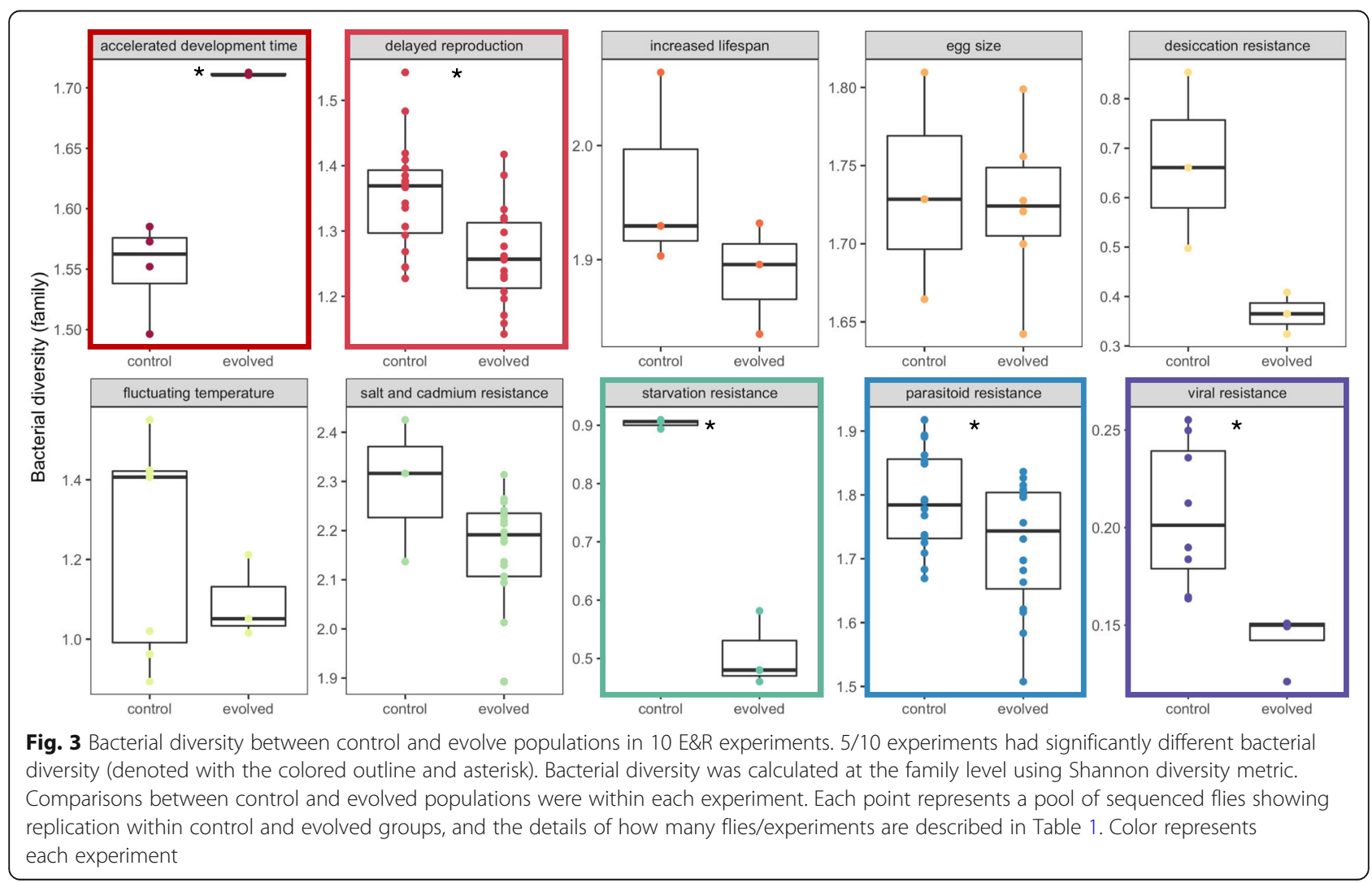


Table 3 Statistical differences between control and evolved microbiomes

\begin{tabular}{llll}
\hline Pressure & test stat & df & significance \\
\hline accelerated development & $\mathrm{t}=-8.116$ & $\mathrm{df}=3.009$ & $p$-value $=0.004$ \\
delayed reproduction & $\mathrm{t}=3.558$ & $\mathrm{df}=33.652$ & $p$-value $=0.001$ \\
increased lifespan & $\mathrm{t}=1.364$ & $\mathrm{df}=3.172$ & $p$-value $=0.261$ \\
egg size & $\mathrm{t}=0.213$ & $\mathrm{df}=3.105$ & $p$-value $=0.845$ \\
desiccation resistance & $\mathrm{t}=2.883$ & $\mathrm{df}=2.224$ & $p$-value $=0.090$ \\
fluctuating temperature & $\mathrm{t}=1.238$ & $\mathrm{df}=7.996$ & $p$-value $=0.251$ \\
salt and cadmium resistance & $\mathrm{t}=1.431$ & $\mathrm{df}=2.287$ & $p$-value $=0.274$ \\
starvation resistance & $\mathrm{t}=10.448$ & $\mathrm{df}=2.065$ & $p$-value $=0.008$ \\
parasitoid resistance & $\mathrm{t}=2.179$ & $\mathrm{df}=28.394$ & $p$-value $=0.038$ \\
viral resistance & $\mathrm{t}=4.265$ & $\mathrm{df}=9.829$ & $p$-value $=0.002$ \\
\hline
\end{tabular}

inconsistent. One study found that the microbiome was significantly different when flies were shifted to a high fat diet, but not when starved [47], while another study also found no differences when shifted to low or high sugar diets [48] within their lifespan. Finally, in flies mono-associated with Lactobacillus reared in nutrient poor diets, Lactobacillus evolved beneficial mutations that promoted fly growth in only 5 fly generations [49]. While most experiments did not manipulate diets in our E\&R analysis, the findings from these three studies suggest a range of timescales in which the microbiome may evolve, though few studies have actually collected controlled time series data on microbiome change in Drosophila. Conducting longitudinal surveys of the
Table 4 Summary statistics of mixed model to assess the relationship between duration of selection and change in diversity. Random effect was modeled as experiment (i.e., accelerated development time, starvation resistance, etc.)

\begin{tabular}{llll}
\hline Fixed effects & & & \\
& Estimate (std. error) & T-value & $\operatorname{Pr}(>|\mathrm{t}|)$ \\
Intercept & $-0.185(0.162)$ & -1.137 & 0.291 \\
log10.length & $0.046(0.097)$ & 0.473 & 0.649 \\
Random effects & & & \\
& Variance & Std. deviation & \\
Experiment & 0.0225 & 0.1500 & \\
Residual & 0.007 & 0.084 & \\
Summary & & & \\
Observations & & 79 & \\
Log Likelihood & & 66.128 & \\
Akaike Inf. Crit. & & -124.256 & \\
Bayesian Inf. Crit. & & -114.779 & \\
\hline
\end{tabular}

microbiome during experimental evolution is essential to understanding if and how the microbiome shapes host evolutionary trajectories.

Wolbachia was found in most of the experiments and often increased in relative abundance in evolved populations (Fig. 5). Wolbachia has a variety of effects on fly biology, ranging from reproductive phenotypes to immunity to nutrition [50-52] and may substantially influence Drosophila evolution [50, 53]. The phenotypic effects exerted by Wolbachia on their hosts often

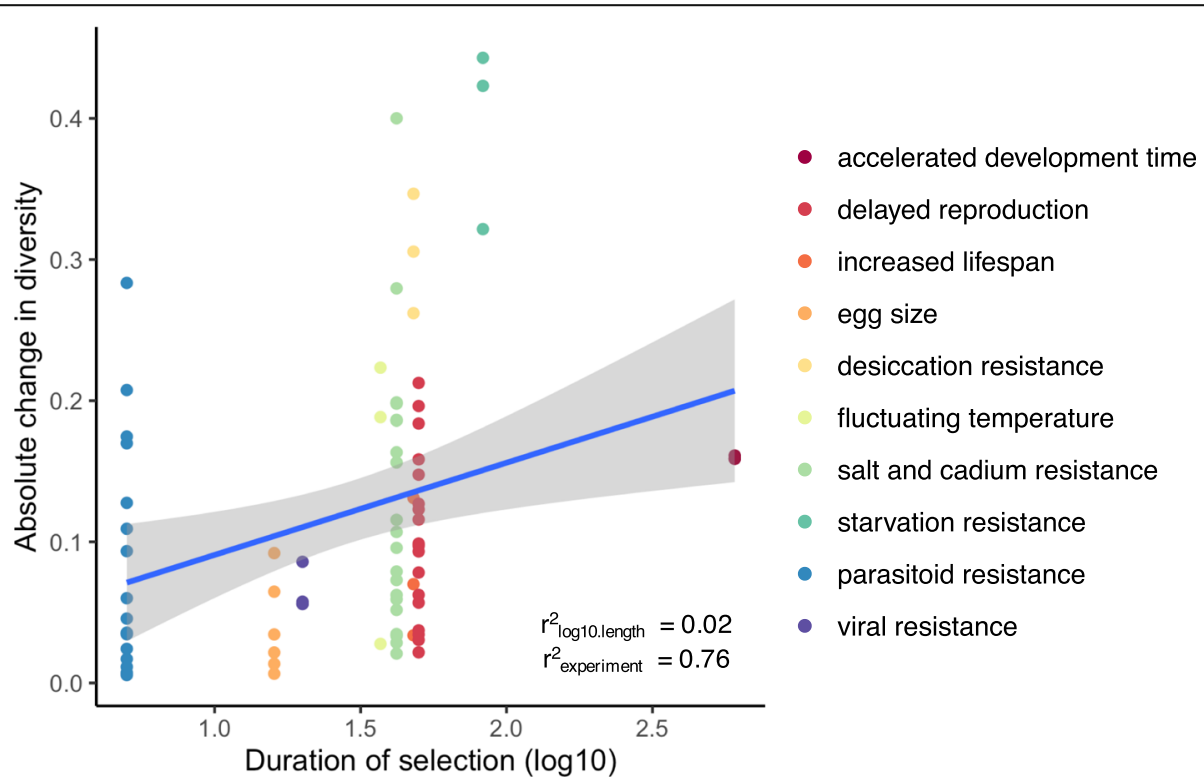

Fig. 4 Evolved bacterial diversity was not significantly correlated with duration of selection after controlling for differences between experiments as random effects $(r=0.05, p=0.649)$. Random effects (experiment) explained $76 \%$ of variation (see Supp. Fig. 11). Generations of selection range from 5 generations (parasitoid resistance) to $>500$ generations (accelerated development). Each point represents the difference between average control diversity and each pool of evolved flies for each experiment. Points are colored by experiment 


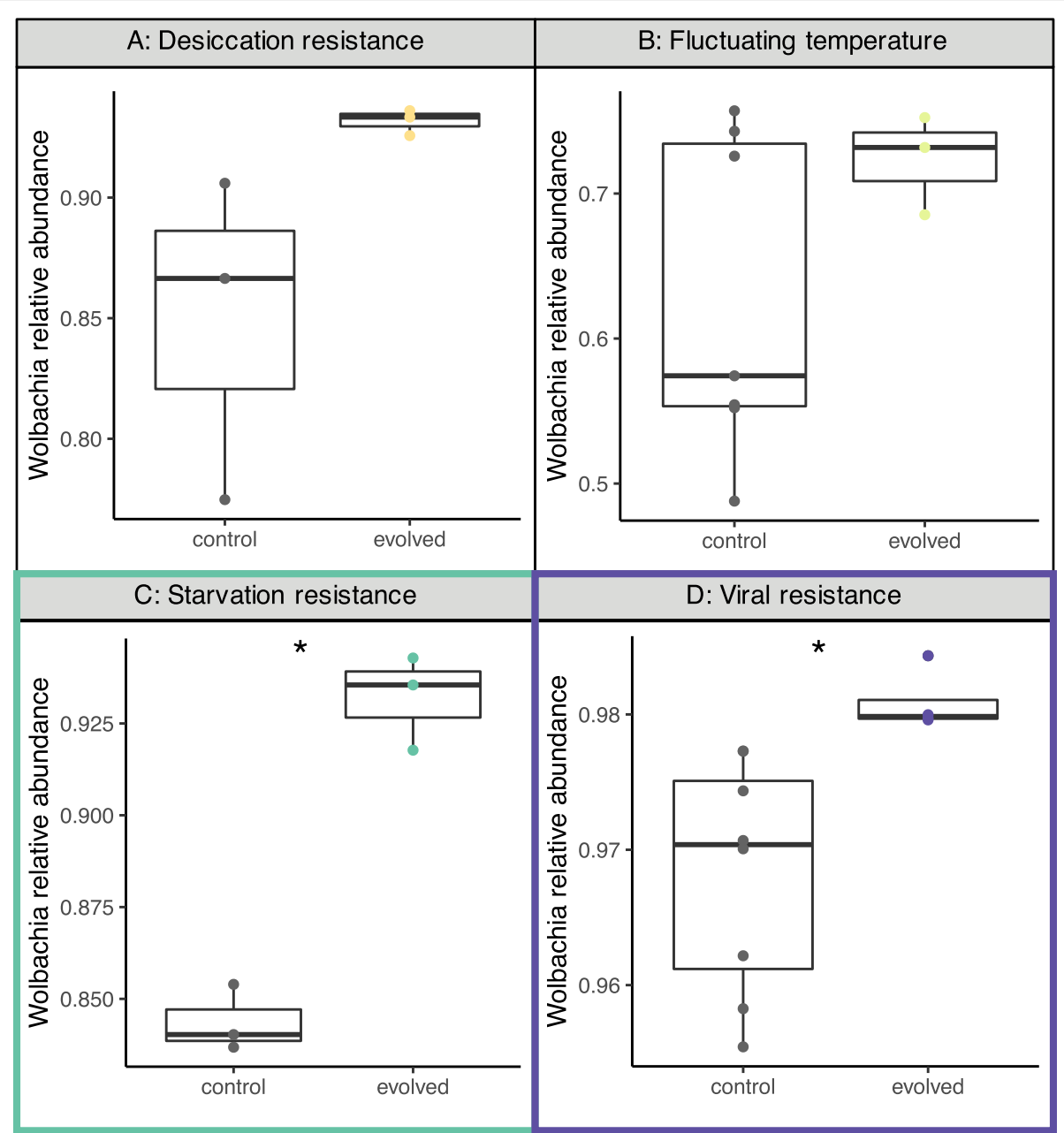

Fig. 5 Wolbachia relative abundance tended to increase in evolved populations for four studies, but was only statistically significant in starvation resistance and viral resistance (denoted with colored box and asterisk). Comparisons were made within each study between control and evolved populations. Each point represents a pool of flies

Table 5 Statistical differences in Wolbachia relative abundance between control (C) and evolved (E) populations

\begin{tabular}{|c|c|c|c|c|c|c|}
\hline Experiment & $\mathrm{C} \min$ & $C \max$ & C avg & E min & $E \max$ & E avg \\
\hline \multicolumn{7}{|l|}{ Desiccation resistance } \\
\hline Relative abundance & 0.775 & 0.906 & 0.849 & 0.926 & 0.936 & 0.932 \\
\hline \multicolumn{7}{|c|}{ Kruskal-Wallis $X^{2}=3.8571, \mathrm{df}=1, p$-value $=0.04953$} \\
\hline \multicolumn{7}{|c|}{ Fluctuating temperature } \\
\hline Relative abundance & 0.488 & 0.757 & 0.628 & 0.685 & 0.752 & 0.723 \\
\hline \multicolumn{7}{|c|}{ Kruskal-Wallis $X^{2}=1.0519, \mathrm{df}=1, p$-value $=0.3051$} \\
\hline \multicolumn{7}{|l|}{ Starvation resistance } \\
\hline Relative abundance & 0.837 & 0.854 & 0.844 & 0.918 & 0.943 & 0.932 \\
\hline \multicolumn{7}{|c|}{ Kruskal-Wallis $X^{2}=3.8571, \mathrm{df}=1, p$-value $=0.04953$} \\
\hline \multicolumn{7}{|l|}{ Viral resistance } \\
\hline Relative abundance & 0.955 & 0.977 & 0.968 & 0.98 & 0.984 & 0.981 \\
\hline \multicolumn{7}{|c|}{ Kruskal-Wallis $X^{2}=7.3846, \mathrm{df}=1, p$-value $=0.006578$} \\
\hline
\end{tabular}

depend on the degree of increase in abundance. For example, higher abundance provided stronger cytoplasmic incompatibilities [54], increased protection from viruses [55], or greater reductions in lifespan [56]. However, several factors may actually confound the Wolbachia results observed in our meta-analysis here. Infection was only assessed from pools of flies - we do not have access to individual level status (Table 1). Relative abundance may reflect the average relative abundance within individuals or heterogeneous infection patterns across individuals. We believe this second scenario of heterogeneous infection across individuals is not likely for the four high Wolbachia studies we examined in more detail. In a study that examined how Wolbachia infection spreads within outbred populations, Wolbachia infection across individuals increased to $80 \%$ by 10 generations and nearly $100 \%$ by 32 generations [57]. This suggests that for the four high Wolbachia studies, most individuals 
were infected. However, for the three studies with low Wolbachia abundance, it may be that Wolbachia infection status is highly heterogeneous across individuals or reflects recent Wolbachia infections (or simply contamination). Fly age also affects Wolbachia abundance, increasing in older flies [58]. Unfortunately, age of flies was only described in $3 / 10$ studies (Table 1 ), but was always similar between control and evolved populations. Age might affect our results if control and evolved populations were systematically collected at different ages. We think this unlikely as it would also bias the genomic analysis as survival may differ between control and evolved populations at different ages (e.g., viability selection). Overall, designing experiments that explicitly control for Wolbachia infection is necessary to understand its potential influence on host-microbiome evolution $[14,23]$.

Wolbachia may also interact and change competitive interactions within the microbiome. In a comparison of a single genotype of flies infected and uninfected with Wolbachia, uninfected flies had twice as much Acetobacter [59]. However, in the same study, a different fly genotype did not display this effect. Yet, another study found that Wolbachia infection increased Acetobacter abundance [60]. These effects are inconsistent and likely depend on interactions between fly genotype, Wolbachia genotype, and environmental conditions. If Wolbachia interacts positively or negatively with different bacteria, then Wolbachia may also influence how the microbiome shapes host phenotypes and contributes to the host evolutionary trajectory.

Wolbachia may be more closely linked to the host evolutionary trajectory because it is vertically transmitted, while the rest of the Drosophila microbiome is environmentally acquired. The joint evolutionary trajectory with the host may change the response to selection in vertically transmitted microbes, like Wolbachia, compared to environmentally acquired microbes $[5,6]$. Taken together, the interactions between Wolbachia, host, and microbiome are likely complicated. We note that computationally removing Wolbachia reads leads to differences in estimates for diversity, where sometimes diversity increases or decreases between control and evolved populations (Supp. Fig. 12). Manipulative experiments clearing Wolbachia infections and comparing the response in both the microbiome and host selection response would show if and how Wolbachia contributes to host evolution. $\sim 50 \%$ of all arthropod species are predicted to be infected with Wolbachia or similar intracellular symbionts [61], and these microbe-microbe interactions may have important implications for the host [62].

While this is the first examination of the microbiome in the E\&R context, other studies have implicated the microbiome in host adaptation in D. melanogaster. For example, as previously mentioned, when flies were monoassociated with Lactobacillus plantarum in nutrient poor environments, L. plantarum rapidly evolved symbiotic benefits to increase fly fitness [49]. Across replicates, the de novo appearance of several SNPs in the acetate kinase gene $(a c k A)$ in $L$. plantarum promoted larval growth and nutrition, and subsequently, this $L$. plantarum variant increased in frequency across fly generations. In another study, microbiome manipulation shifted allele frequency in seasonally evolving $D$. melanogaster to match latitudinal patterns of fly genetics [63]. Both of these studies rely on mono-associations with single microbes, but this likely does not realistically capture host-microbe dynamics. Higher order interactions among bacteria shape phenotypes in Drosophila [45, 46]. Interaction among microbes, like cross-feeding of metabolites between Acetobacter and Lactobacillus, can enable mutually beneficial growth for both bacteria species as well as increases bacterial growth, but critically also alters fly metabolism [64]. This suggests that mutations within bacterial species may affect interactions across bacteria in the microbiome. Furthermore, even strainlevel variation within a bacterial species can have divergent effects on host phenotypes $[65,66]$. The technical challenges associated with accurately quantifying genetic variation across complex microbial populations necessitated these mono-association experiments. Fortunately, new emerging methods are enabling the identification of signatures of selection in complex microbiomes $[67,68]$. Future experiments with more complex and realistic microbiomes will show how microbe-microbe interactions contribute to host adaptation.

Taken together with our analyses, as the host evolves, the microbiome frequently changes in response to host selection. More generally, other systems like Brassica and Arabidopsis have also shown that selection on hosts changes the microbiome as well $[69,70]$. In both these studies, transplanting an evolved microbiome into unevolved hosts changed host phenotypes, suggesting that the microbiome has the capacity to transfer adaptive potential. Similar approaches could be applied to Drosophila following E\&R experiments. Importantly, our study here only characterized change in microbial community composition, but not how specific bacteria evolved (e.g., mutations or polymorphisms) in response to host selection. New computational and sequencing techniques that enable variant discovery in bacteria combined with longitudinal sampling have quantified eco-evolutionary dynamics in mammalian microbiomes to show that bacteria frequently acquire new mutations to increase fitness to respond to biotic fluctuations in the gut environment [71, 72]. Combined with the rich genetic resources and experimental ease in Drosophila, 
microbiome transplants and novel computation techniques will illuminate key processes underlying hostmicrobiome evolution.

We note the experiments analyzed here were not designed explicitly to test the role of the microbiome in host adaptation. This may impact our results in several ways. None of these studies were executed with quality control measures that can affect estimates of microbial diversity, such as process blanks during DNA extraction, no template controls during PCR, and batch effects during library preparation [67, 73-75]. While we applied an arbitrary cutoff to remove contaminants, it is difficult to know how potential contaminants may affect the observed results. However, contamination would have to differentially affect control and evolved microbiomes to influence our results--which we believe is unlikely. Surveys of microbial diversity in $D$. melanogaster typically use 16S rRNA profiling and find bacteria from the Acetobacteraceae, Firmicutes, and Enterobacteriaceae [22, 24, 76]. Our mapping approach detected these bacteria commonly associated with $D$. melanogaster, but also found abundant methanogens and human commensal microbes (Fig. 1). One discrepancy could arise from our metagenomic approach, which will often lead to different conclusions than 16S rRNA profiling [67]. Mining metagenomes from existing whole genome sequencing is an emerging area of research in the microbiome, and more work is necessary for biological interpretations [5, 67]. Finally, none of the flies sequenced in these studies were surface sterilized, and thus, the metagenomes characterized here result from both the external body surface and internal gut microbiome. However, the external microbiome is orders of magnitude less abundant than the internal microbiome across the fly lifespan [77]. While we cannot distinguish between external and internal microbiomes in this analysis, future studies should be clear if the total (external and internal) or gut microbiomes were sequenced. Nevertheless, the consistent differences in the microbiome across experiments shown here highlight how E\&R experiments could provide exemplary opportunities to investigate the genetic basis underlying host-microbiome evolution.

\section{Conclusions}

For researchers interested in adapting the E\&R approach for host-microbiome interactions, we have several key recommendations. As we have suggested above, more intensive temporal sampling to capture both microbial and host evolution is necessary. For Drosophila-microbiome E\&R experiments, researchers may wish to begin the experimental evolution by standardizing the microbiome between control and evolved populations, like with the 5 -species bacterial community commonly used $[23,78]$ or fly feces to mimic natural, but standardized microbial inoculation [79, 80]. Second, as much of the microbiome is determined by the environment in flies, researchers need to use consistent brands of yeast, preservatives, and other aspects of diet/environment. For example, different preservatives have different effects on the microbiome and behavioral traits [40, 41]. Drosophila in different labs in the same building (with the same kitchen for fly food) had different microbiomes [76], suggesting that several aspects of the environment are important in shaping the fly microbiome. Finally, as outlined by Goodrich et al. [81], microbiome research requires careful planning (with both biological and technical controls), extensive documentation, and consistency. Importantly, we are not advocating that every E\&R experiment incorporates the microbiome, but note that the microbiome may impact conclusions from E\&R experiments. For researchers not explicitly interested in the microbiome, our primary recommendation is to clear fly lines of Wolbachia to avoid potential confounding effects between host genetic and Wolbachia evolution as others have suggested [14, 23].

In conclusion, the microbiome frequently responded to selection in ten E\&R studies in D. melanogaster. Our results here associate the microbiome in the host response to some selective pressures, but more work is necessary to partition the relative effects of host genetics and microbial evolution. We observed large differences in bacterial diversity between control and evolved populations, but a key question remains--if and how the microbiome alters the host response to selection. Combining E\&R experiments with approaches from quantitative genetics will be especially fruitful to dissecting the microbial contribution to host evolution [6]. Tracking the rate of microbial evolution over multiple timepoints during fly adaptation will be particularly helpful to elucidate whether the microbiome shapes the host evolutionary trajectory. Partitioning the microbial effects on host phenotype during adaptation may show that microbiome facilitates or impedes host adaptation. Reciprocal transplants over the course of host adaptation will also demonstrate how the microbiome modifies host evolution. Our results here suggest that the microbiome might influence host evolution, but do not prove it. To measure how the microbiome affects host evolutionary trajectory, combining several of these techniques will be necessary. Overall, incorporating the microbiome into E\&R experiments will provide fundamental insights into hostmicrobiome evolution.

\section{Methods}

We searched the literature for E\&R experiments in $D$. melanogaster where replicated control and selection lines were derived from outbred populations and raw .fastq data were publicly available. We found 10 studies 
that met these criteria. Our analyses captured a wide range of different selection pressures, from life-history traits to abiotic and pathogen pressures, enabling generalizations about the microbiome response to host selection. In all cases, the E\&R approach sequenced pools of individuals from different selection regimes, but each E\&R study had different levels of replication (summarized in Table 1). We report the diet as described in the publication for each study (Table 1). While most studies did not publish specifics about the diet, we noted the diets that differed between control and evolved populations (only one study [31]); if the publication did not specify, we reasonably assumed diets were the same. These were the only data available from published E\&R experiments in D. melanogaster at the time of submission.

Raw sequences were cleaned using Trimmomatic [82] to remove sequencing adapters, remove low quality reads (average quality per base $>15$ ), and drop reads shorter than 20 bases long. Then, bacterial reads were assigned at the family level using Kraken [83]. Relative abundance of bacterial families were determined using Bracken [84]. We removed any low abundance bacterial family that was assigned fewer than 100 reads as potential contaminants.

Bacterial data was analyzed using the phyloseq package [85]. To assess if bacterial communities were fully sampled, rarefaction was performed (step size $=1000$ ) using ggrare [86]. Rarefaction curves indicate communities were fully sampled in all experiments (Supp. Figs 1-10). Beta-diversity to test differences in community composition between control and evolved populations was performed using PERMANOVA on Jaccard similarity. Bacterial alpha-diversity was calculated using the Shannon diversity index. For each experiment, each line was subsampled with replacement to the minimum number of reads in the experiment. Diversity was calculated on this rarefied library. The subsampling was performed 100 times to minimize stochasticity and artificial inflation of diversity associated with rarefaction [87]. Diversity was then averaged across the 100 subsampling efforts and compared between control and evolved lines. We determined significance using Welch's t-test. We then tested whether two factors were sufficient to explain variation in microbial diversity between control and evolved lines: duration of selection (i.e., the number of fly generations) and Wolbachia infection.

First, the duration of selection ranged from 5 to 605 generations. We reasoned that selection response in the microbiome might be influenced by length of selection (the longer the selection, the more divergent the microbiome between control and evolved lines). To test if the duration of selection was correlated with changes in microbial diversity, we first calculated the average microbial diversity for the control lines. We then subtracted the diversity of each evolved line from the averaged control diversity to calculate change in diversity. Because we had positive and negative changes in diversity, we used the absolute difference. We performed a linear regression between change in diversity and the $\log 10$ duration of selection, modeled as $\mathrm{Y}=\mathrm{a}+\mathrm{b}+\mathrm{e}$, where $\mathrm{Y}=$ change in diversity, $\mathrm{a}=\log 10$ duration of selection, $\mathrm{b}=$ random effect of experiment, and $\mathrm{e}=$ residual error. Lme4 was used to perform the regression in $\mathrm{R}$ [88].

Given that Wolbachia reads frequently make up the majority of the microbial reads (Supp. Table 1), we examined if Wolbachia relative abundance differed between control and evolved populations. We focused on only the four studies with Wolbachia relative abundance $>2 \%$. Statistical significance was assessed with a Kruskal-Wallis test on Wolbachia relative abundance.

\section{Abbreviations}

E\&R: Evolve and Resequence; IIS: Insulin/insulin-like signalling

\section{Supplementary Information}

The online version contains supplementary material available at https://doi. org/10.1186/s12866-021-02168-4.

Additional file 1: Supp. Table 1. Relative abundance of Wolbachia in each of the 10 E\&R experiments

Additional file 2: Supp. Table 2. List of accession numbers for raw genomic data from the 10 E\&R experiments

Additional file 3: Supp. Table 3. Statistical differences between control and evolved microbiomes with Wolbachia reads computationally removed

Additional file 4: Supp. Figures 1-10. Distribution of sequencing depth, rarefaction curves, and relative abundance of bacteria (family-level) for each of the 10 E\&R experiments

Additional file 5: Supp. Figure 11. Estimates of random effects (i.e., E\&R experiment) from linear model to test the correlation between microbial change and duration of selection

Additional file 6: Supp. Fig. 12. Differences in bacterial diversity when including (full bacterial community) or excluding Wolbachia reads in four of the E\&R experiments analyzed

\section{Acknowledgements}

We thank members of the Ayroles lab for helpful feedback

\section{Authors' contributions}

$\mathrm{LPH}$ and JFA designed research. LPH collected, analyzed data and wrote the manuscript with JFA contributing to revisions. All authors read and approved the manuscript.

\section{Funding}

LPH was supported by NSF-GRFP under grant DGE1656466 and National Institutes of Health (NIH) grant GM124881 to JFA. Funding agencies were not involved in the design, collection, analysis, interpretation or writing of this manuscript.

Availability of data and materials

The raw fastq data are available in the NCBI Sequence Read Archive, and accession numbers can be found in Supp. Table 2 . The code used to analyze data can be found at https://github.com//phenry/extgeno_e-r. 


\section{Declarations}

\section{Ethics approval and consent to participate}

Not applicable.

\section{Consent for publication}

Not applicable.

\section{Competing interests}

The authors declare that they have no competing interests.

\section{Author details}

'Department of Ecology \& Evolutionary Biology, 150 Carl Icahn Laboratory, Princeton University, Princeton, NJ 08544, USA. ² Lewis-Sigler Institute for Integrative Genomics, Princeton University, Princeton, NJ 08544, USA.

Received: 15 July 2020 Accepted: 31 March 2021

Published online: 09 April 2021

\section{References}

1. Turnbaugh PJ, Ley RE, Hamady M, Fraser-Liggett CM, Knight R, Gordon J. The human microbiome project. Nature. 2007;449:804-10.

2. Friesen ML, Porter SS, Stark SC, von Wettberg EJ, Sachs JL, Martinez-Romero E. Microbially Mediated Plant Functional Traits. Annu Rev Ecol Evol Syst. 2011:42:23-46

3. McFall-Ngai M, Hadfield MG, Bosch TC, Carey HV, Domazet-Loso T, Douglas $A E$, et al. Animals in a bacterial world, a new imperative for the life sciences. Proc Natl Acad Sci. 2013;110. https://doi.org/10.1073/pnas.1218525110.

4. Moran NA, Sloan DB. The hologenome concept: Helpful or hollow? PLoS Biol. 2015;13:e1002311.

5. Koskella B, Hall LJ, Metcalf CJE. The microbiome beyond the horizon of ecological and evolutionary theory. Nat Ecol Evol. 2017;1:1606-15.

6. Henry LP, Bruijning M, Forsberg SKG, Ayroles JF. Can the microbiome influence host evolutionary trajectories? bioRxiv. 2019:700237.

7. Ferreiro A, Crook N, Gasparrini AJ, Dantas G. Multiscale Evolutionary Dynamics of Host-Associated Microbiomes. Cell. 2018:172:1216-27.

8. Hurst GDD. Extended genomes: symbiosis and evolution. Interface Focus. 2017;7:20170001.

9. Carthey AJR, Gillings MR, Blumstein DT. The Extended Genotype: Microbially Mediated Olfactory Communication. Trends Ecol Evol. 2018;33:885-94.

10. Mueller UG, Sachs JL. Engineering Microbiomes to Improve Plant and Animal Health. Trends Microbiol. 2015;23:606-17.

11. Hoang KL, Morran LT, Gerardo NM. Experimental Evolution as an Underutilized Tool for Studying Beneficial Animal-Microbe Interactions. Front Microbiol. 2016:07. https://doi.org/10.3389/fmicb.2016.01444.

12. Kofler $\mathrm{R}$, Schlötterer $C$. A guide for the design of evolve and resequencing studies. Mol Biol Evol. 2014;31:474-83.

13. Long A, Liti G, Luptak A, Tenaillon O. Elucidating the molecular architecture of adaptation via evolve and resequence experiments. Nat Rev Genet. 2015; 16:567-82.

14. Schlötterer C, Kofler R, Versace E, Tobler R, Franssen SU. Combining experimental evolution with next-generation sequencing: a powerful tool to study adaptation from standing genetic variation. Heredity. 2015;114:43140

15. Starr DJ, Cline TW. A host-parasite interaction rescues Drosophila oogenesis defects. Nature. 2002:418:76-9.

16. Clark ME, Anderson CL, Cande J, Karr TL. Widespread prevalence of wolbachia in laboratory stocks and the implications for Drosophila research. Genetics. 2005;170:1667-75

17. Ikeya T, Broughton S, Alic N, Grandison R, Partridge L. The endosymbiont Wolbachia increases insulin/IGF-like signalling in Drosophila. Proc R Soc Lond B Biol Sci. 2009;276:3799-807.

18. Tsuchida T, Koga R, Horikawa M, Tsunoda T, Maoka T, Matsumoto S, et al. Symbiotic bacterium modifies aphid body color. Science. 2010;330:1102-4

19. Difford GF, Plichta DR, Løvendahl P, Lassen J, Noel SJ, Højberg O, et al. Host genetics and the rumen microbiome jointly associate with methane emissions in dairy cows. PLoS Genet. 2018;14:e1007580.

20. Buitenhuis B, Lassen J, Noel SJ, Plichta DR, Sørensen P, Difford GF, et al. Impact of the rumen microbiome on milk fatty acid composition of Holstein cattle. Genet Sel Evol. 2019;51:23.
21. Camarinha-Silva A, Maushammer M, Wellmann R, Vital M, Preuss S, Bennewitz J. Host Genome Influence on Gut Microbial Composition and Microbial Prediction of Complex Traits in Pigs. Genetics. 2017;206:1637-44.

22. Broderick NA, Lemaitre B. Gut-associated microbes of Drosophila melanogaster. Gut Microbes. 2012;3:307-21.

23. Douglas AE. The Drosophila model for microbiome research. Lab Anim. 2018;47:157-64.

24. Walters AW, Hughes RC, Call TB, Walker CJ, Wilcox H, Petersen SC, et al. The microbiota influences the Drosophila melanogaster life history strategy. Mol Ecol. 2019. https://doi.org/10.1111/mec.15344

25. Burke MK, Dunham JP, Shahrestani P, Thornton KR, Rose MR, Long AD. Genome-wide analysis of a long-term evolution experiment with Drosophila. Nature. 2010:467:587-90.

26. Remolina SC, Chang PL, Leips J, Nuzhdin SV, Hughes KA. Genomic basis of aging and life-history evolution in Drosophila melanogaster. Evolution. 2012 66:3390-403.

27. Michalak P, Kang L, Sarup PM, Schou MF, Loeschcke V. Nucleotide diversity inflation as a genome-wide response to experimental lifespan extension in Drosophila melanogaster. BMC Genomics. 2017;18. https://doi.org/10.1186/ s12864-017-3485-0.

28. Jha AR, Miles CM, Lippert NR, Brown CD, White KP, Kreitman M. WholeGenome Resequencing of Experimental Populations Reveals Polygenic Basis of Egg-Size Variation in Drosophila melanogaster. Mol Biol Evol. 2015:32: 2616-32.

29. Kang L, Aggarwal DD, Rashkovetsky E, Korol AB, Michalak P. Rapid genomic changes in Drosophila melanogaster adapting to desiccation stress in an experimental evolution system. BMC Genomics. 2016;17. https://doi.org/1 0.1186/s12864-016-2556-y.

30. Orozco-terWengel P, Kapun M, Nolte V, Kofler R, Flatt T, Schlötterer C. Adaptation of Drosophila to a novel laboratory environment reveals temporally heterogeneous trajectories of selected alleles. Mol Ecol. 2012;21:4931-41.

31. Huang Y, Wright SI, Agrawal AF. Genome-wide patterns of genetic variation within and among alternative selective regimes. PLoS Genet. 2014;10: e1004527.

32. Hardy CM, Burke MK, Everett LJ, Han MV, Lantz KM, Gibbs AG. GenomeWide Analysis of Starvation-Selected Drosophila melanogaster: A Genetic Model of Obesity. Mol Biol Evol. 2018;35:50-65.

33. Jalvingh KM, Chang PL, Nuzhdin SV, Wertheim B. Genomic changes under rapid evolution: selection for parasitoid resistance. Proc R Soc B Biol Sci. 2014;281:20132303.

34. Martins NE, Faria VG, Nolte V, Schlotterer C, Teixeira L, Sucena E, et al. Host adaptation to viruses relies on few genes with different cross-resistance properties. Proc Natl Acad Sci. 2014;111:5938-43.

35. Lesperance DNA, Broderick NA. Meta-analysis of Diets Used in Drosophila Microbiome Research and Introduction of the Drosophila Dietary Composition Calculator (DDCC). G3. 2020;10:2207-11.

36. Shin SC, Kim S-H, You H, Kim B, Kim AC, Lee K-A, et al. Drosophila microbiome modulates host developmental and metabolic homeostasis via insulin signaling. Science. 2011:334:670-4.

37. Chaston JM, Newell PD, Douglas AE. Metagenome-wide association of microbial determinants of host phenotype in Drosophila melanogaster. MBio. 2014;5:e01631-14

38. White KM, Matthews MK, Hughes RC, Sommer AJ, Griffitts JS, Newell PD, et al. A metagenome-wide association study and arrayed mutant library confirm Acetobacter lipopolysaccharide genes are necessary for association with Drosophila melanogaster. G3. 2018;8:1119-27.

39. Selkrig J, Mohammad F, Ng SH, Chua JY, Tumkaya T, Ho J, et al. The Drosophila microbiome has a limited influence on sleep, activity, and courtship behaviors. Sci Rep. 2018;8:10646.

40. Leftwich PT, Clarke NVE, Hutchings MI, Chapman T. Reply to Obadia et al.: Effect of methyl paraben on host-microbiota interactions in Drosophila melanogaster. Proc Natl Acad Sci U S A. 2018;115:E4549-50.

41. Obadia B, Keebaugh ES, Yamada R, Ludington WB, Ja WW. Diet influences host-microbiota associations in Drosophila. Proc Natl Acad Sci U S A. 2018. 115:E4547-8.

42. Sannino DR, Dobson AJ, Edwards K, Angert ER, Buchon N. The Drosophila melanogaster Gut Microbiota Provisions Thiamine to Its Host. MBio. 2018;9 https://doi.org/10.1128/mBio.00155-18.

43. Moran NA, Ochman H, Hammer TJ. Evolutionary and Ecological Consequences of Gut Microbial Communities. Annu Rev Ecol Evol Syst. 2019. https://doi.org/10.1146/annurev-ecolsys-110617-062453. 
44. Dobson AJ, Chaston JM, Newell PD, Donahue L, Hermann SL, Sannino DR, et al. Corrigendum: Host genetic determinants of microbiota-dependent nutrition revealed by genome-wide analysis of Drosophila melanogaster. Nat Commun. 2015;6:7296.

45. Newell PD, Douglas AE. Interspecies Interactions Determine the Impact of the Gut Microbiota on Nutrient Allocation in Drosophila melanogaster. App Environ Microbiol. 2013;80:788-96.

46. Gould AL, Zhang V, Lamberti L, Jones EW, Obadia B, Korasidis N, et al. Microbiome interactions shape host fitness. Proc Natl Acad Sci U S A. 2018; 115:E11951-60.

47. Fink C, Staubach F, Kuenzel S, Baines JF, Roeder T. Noninvasive Analysis of Microbiome Dynamics in the Fruit Fly Drosophila melanogaster. Appl Environ Microbiol. 2013:79:6984-8.

48. Jehrke L, Stewart FA, Droste A, Beller M. The impact of genome variation and diet on the metabolic phenotype and microbiome composition of Drosophila melanogaster. Sci Rep. 2018;8:6215.

49. Martino ME, Joncour $\mathrm{P}$, Leenay $\mathrm{R}$, Gervais $\mathrm{H}$, Shah $\mathrm{M}$, Hughes $\mathrm{S}$, et al. Bacterial Adaptation to the Host's Diet Is a Key Evolutionary Force Shaping Drosophila-Lactobacillus Symbiosis. Cell Host Microbe. 2018;24:109-19.e6.

50. Harcombe W, Hoffmann AA. Wolbachia effects in Drosophila melanogaster: In search of fitness benefits. J Invertebr Pathol. 2004;87:45-50.

51. Teixeira L, Ferreira Á, Ashburner M. The bacterial symbiont Wolbachia induces resistance to RNA viral infections in Drosophila melanogaster. PLoS Biol. 2008;6:e1000002.

52. Ponton F, Wilson K, Holmes A, Raubenheimer D, Robinson KL, Simpson SJ. Macronutrients mediate the functional relationship between Drosophila and Wolbachia. Proc R Soc B Biol Sci. 2015;282. https://doi.org/10.1098/rspb.2 014.2029.

53. Fry AJ, Rand DM, Poulin R. Wolbachia interactions that determine drosophila melanogaster survival. Evolution. 2002;56:1976-81.

54. Clancy DJ, Hoffmann AA. Environmental effects on cytoplasmic incompatibility and bacterial load in Wolbachia-infected Drosophila simulans. Entomol Exp Appl. 1998;86:13-24.

55. Chrostek E, Marialva MSP, Esteves SS, Weinert LA, Martinez J, Jiggins FM, et al. Wolbachia Variants Induce Differential Protection to Viruses in Drosophila melanogaster: A Phenotypic and Phylogenomic Analysis. PLoS Genet. 2013;9:e1003896.

56. Martinez J, Ok S, Smith S, Snoeck K, Day JP, Jiggins FM. Should symbionts be nice or selfish? Antiviral effects of Wolbachia are costly but reproductive parasitism is not. PLoS Pathog. 2015;11:e1005021.

57. Kriesner P, Hoffmann AA. Rapid spread of a Wolbachia infection that does not affect host reproduction in Drosophila simulans cage populations. Evolution. 2018. https://doi.org/10.1111/evo.13506.

58. Kaur R, Martinez J, Rota-Stabelli O, Jiggins FM, Miller WJ. Age, tissue, genotype and virus infection regulate Wolbachia levels in Drosophila. Mol Ecol. 2020;29:2063-79.

59. Simhadri RK, Fast EM, Guo R, Schultz MJ, Vaisman N, Ortiz L, et al. The Gut Commensal Microbiome of Drosophila melanogaster Is Modified by the Endosymbiont Wolbachia. mSphere. 2017;2. https://doi.org/10.1128/ msphere.00287-17.

60. Ye YH, Seleznev A, Flores HA, Woolfit M, McGraw EA. Gut microbiota in Drosophila melanogaster interacts with Wolbachia but does not contribute to Wolbachia-mediated antiviral protection. J Invertebr Pathol. 2017;143:1825.

61. Weinert LA, Araujo-Jnr EV, Ahmed MZ, Welch JJ. The incidence of bacterial endosymbionts in terrestrial arthropods. Proc Biol Sci. 2015;282:20150249.

62. Brinker P, Fontaine MC, Beukeboom LW, Falcao Salles J. Host, Symbionts, and the Microbiome: The Missing Tripartite Interaction. Trends Microbiol. 2019;27:480-8

63. Rudman SM, Greenblum S, Hughes RC, Rajpurohit S, Kiratli O, Lowder DB, et al. Microbiome composition shapes rapid genomic adaptation of Drosophila melanogaster. Proc Natl Acad Sci U S A. 2019;116:20025-32.

64. Sommer AJ, Newell PD. Metabolic basis for mutualism between gut bacteria and its impact on the Drosophila melanogaster host. Appl Environ Microbiol. 2019; https://aem.asm.org/content/85/2/e01882-18.abstract.

65. Obadia B, Güvener ZT, Zhang V, Ceja-Navarro JA, Brodie EL, Ja WW, et al Probabilistic Invasion Underlies Natural Gut Microbiome Stability. Curr Biol. 2017:27:1999-2006.e8

66. Douglas AE. Contradictory Results in Microbiome Science Exemplified by Recent Drosophila Research. mBio. 2018;9. https://doi.org/10.1128/mBio.01 758-18.
67. Knight R, Vrbanac A, Taylor BC, Aksenov A, Callewaert C, Debelius J, et al. Best practices for analysing microbiomes. Nat Rev Microbiol. 2018;16:410-22.

68. Garud NR, Pollard KS. Population Genetics in the Human Microbiome. Trends Genet. 2020;36:53-67.

69. Lau JA, Lennon JT. Rapid responses of soil microorganisms improve plant fitness in novel environments. Proc Natl Acad Sci U S A. 2012;109:14058-62.

70. Panke-Buisse K, Poole AC, Goodrich JK, Ley RE, Kao-Kniffin J. Selection on soil microbiomes reveals reproducible impacts on plant function. ISME J. 2015:9:980-9.

71. Garud NR, Good BH, Hallatschek O, Pollard KS. Evolutionary dynamics of bacteria in the gut microbiome within and across hosts. PLoS Biol. 2019;17: e3000102.

72. Barroso-Batista J, Pedro MF, Sales-Dias J, Pinto CJG, Thompson JA, Pereira H, et al. Specific Eco-evolutionary Contexts in the Mouse Gut Reveal Escherichia coli Metabolic Versatility. Curr Biol. 2020:30:1049-62.e7.

73. Lauder AP, Roche AM, Sherrill-Mix S, Bailey A, Laughlin AL, Bittinger K, et al. Comparison of placenta samples with contamination controls does not provide evidence for a distinct placenta microbiota. Microbiome. 2016;4:29.

74. Pollock J, Glendinning L, Wisedchanwet T, Watson M. The Madness of Microbiome: Attempting To Find Consensus "Best Practice" for $16 \mathrm{~S}$ Microbiome Studies. Appl Environ Microbiol. 2018;84:e02627-17.

75. de Goffau MC, Lager S, Sovio U, Gaccioli F, Cook E, Peacock SJ, et al. Human placenta has no microbiome but can contain potential pathogens. Nature. 2019:572:329-34

76. Chandler JA, Lang JM, Bhatnagar S, Eisen JA, Kopp A. Bacterial communities of diverse Drosophila species: ecological context of a host-microbe model system. PLoS Genet. 2011;7:e1002272.

77. Ren C, Webster P, Finkel SE, Tower J. Increased Internal and External Bacterial Load during Drosophila Aging without Life-Span Trade-Off. Cell Metab. 2007;6:144-52

78. Koyle ML, Veloz M, Judd AM, Wong AC-N, Newell PD, Douglas AE, et al. J Vis Exp. 2016:e54219. https://doi.org/10.3791/54219.

79. Erkosar B, Kolly S, van der Meer JR, Kawecki TJ. Adaptation to Chronic Nutritional Stress Leads to Reduced Dependence on Microbiota in Drosophila melanogaster. MBio. 2017;8:e01496-17. https://doi.org/10.1128/ mBio.01496-17.

80. Cavigliasso F, Dupuis C, Savary L, Spangenberg JE, Kawecki TJ. Experimental evolution of post-ingestive nutritional compensation in response to a nutrient-poor diet. Proc R Soc B Biol Sci. 2020;287:20202684. https://doi. org/10.1098/rspb.2020.2684.

81. Goodrich JK, Di Rienzi SC, Poole AC, Koren O, Walters WA, Caporaso JG, et al. Conducting a microbiome study. Cell. 2014;158:250-62.

82. Bolger AM, Lohse M, Usadel B. Trimmomatic: a flexible trimmer for Illumina sequence data. Bioinformatics. 2014;30:2114-20.

83. Wood DE, Salzberg SL. Kraken: ultrafast metagenomic sequence classification using exact alignments. Genome Biol. 2014;15:R46.

84. Lu J, Breitwieser FP, Thielen P, Salzberg SL. Bracken: Estimating species abundance in metagenomics data; 2016. https://doi.org/10.1101/051813.

85. McMurdie PJ, Holmes S. phyloseq: an R package for reproducible interactive analysis and graphics of microbiome census data. PLoS One. 2013;8:e61217.

86. Kandlikar GS, Gold ZJ, Cowen MC, Meyer RS, Freise AC, Kraft NJB, et al. ranacapa: An R package and Shiny web app to explore environmental DNA data with exploratory statistics and interactive visualizations. F1000Res. 2018; 7:1734.

87. McMurdie PJ, Holmes S. Waste not, want not: why rarefying microbiome data is inadmissible. PLoS Comput Biol. 2014;10:e1003531.

88. Bates D, Sarkar D, Bates MD, Matrix L. The Ime4 package. R package version. 2007;2:74

\section{Publisher's Note}

Springer Nature remains neutral with regard to jurisdictional claims in published maps and institutional affiliations. 